\title{
Le rôle de la conscience phonologique dans l'apprentissage de la lecture en français langue seconde par les enfants arabophones : comparaison entre lecteurs normaux et dyslexiques
}

\author{
Smail Layes \\ Université El-Oued \\ Kamel Layes \\ Université d'Alger 2 \\ Khenfour Hichem \\ Université El-Oued
}

(Text received August 27 2018; accepted November 16 2018; final version December 25

2018)

DOI: https://doi.org/10.5565/rev/jt13.775

Résumé : Cette étude examine le rôle de la conscience phonologique (CP) en arabe (L1) dans la lecture en français (L2) chez les enfants arabophones. Deux groupes de lecteurs normaux $(n=42)$ et dyslexiques $(n=30)$ ont participé à cette étude, et ont passé des tests de lecture de mots et de pseudo mots ainsi que de CP en L1 et en langue seconde (L2). Les résultats ont révélé des différences statistiquement significatives entre les deux groupes de lecteurs en lecture de mots, de pseudo-mots et dans la CP en L1 et L2. Le décodage de pseudo mots et la CP prédisent significativement la performance de lecture en L2 dans chaque groupe de lecteurs. Les résultats indiquent que la $\mathrm{CP}$ en L1 s'avère un indice tangible de la performance de lecture en français (L2) chez les enfants arabophones. Ces résultats ont été discutés en termes d'éventuel effet de transfert positif des connaissances phonologiques entre L1 et L2.

Mots-clés : lecture en L1 et L2, conscience phonologique, dyslexie, transfert phonologique

\begin{abstract}
This study examined the role of phonological awareness (PA) in reading in French (L2) in Arabic-speaking children. Two groups of typical readers $(n=42)$ and dyslexics $(\mathrm{n}=30)$ participated in this study, and completed word and pseudo-words reading and PA tests in both $\mathrm{L} 1$ and $\mathrm{L} 2$. The results revealed statistically significant differences between typical readers and dyslexics in all measures of word and pseudoword decoding and phonological awareness skills in L1 and L2. Significant correlation coefficients between the different measures were also obtained including all participants. These results indicate that PA in L1 is a noticeable factor of reading performance in French (L2) in Arabic-speaking children. The results are discussed in terms of a potential effect of phonological knowledge positive transfer from Arabic (L1) to learning to read in French (L2).
\end{abstract}

Keywords: reading in L1 and L2, phonological awareness, dyslexia, phonological transfer 
Resumen: Este estudio examinó el papel de la conciencia fonológica en la lectura en francés como segunda lengua (L2) por parte de niños arabófonos. Dos grupos de lectores típicos $(\mathrm{n}=42)$ y disléxicos $(\mathrm{n}=30)$ participaron en este estudio, y completaron la lectura de palabras y pseudopalabras, además de pruebas de conciencia fonológica, tanto en L1 como en L2. Los resultados revelaron diferencias estadísticamente significativas entre lectores típicos y disléxicos en todas las medidas de descodificación de palabras y pseudopalabras, y en las habilidades de conciencia fonológica en L1 y L2. También se obtuvieron coeficientes de correlación significativos entre las diferentes medidas, incluidos todos los participantes. Estos resultados indican que la conciencia fonológica en L1 es un factor notable de rendimiento de lectura en francés (L2) en niños de habla árabe. Los resultados son discutidos en términos de un posible efecto de la transferencia positiva del conocimiento fonológico del árabe (L1) al aprendizaje de la lectura en francés (L2).

Palabras clave: lectura en L1 y L2, conciencia fonológica, dislexia, transferencia fonológica

\section{Introduction}

Bien que de nombreuses définitions de la dyslexie soient proposées, on s'accorde généralement à considérer que la dyslexie développementale est un trouble d'origine neurocognitif qui se caractérise par des difficultés observables et persistantes à apprendre à lire chez un enfant qui, par ailleurs, manifeste des capacités intellectuelles normales, ne présente pas de déficiences sensorielles (visuelle et auditive) et suit une scolarité régulière (DSM-IV, 2000). Plus récemment, Le DSM-5 considère la dyslexie développementale comme trouble faisant partie d'un trouble plus général dénommé les «troubles spécifiques d'apprentissage » (TSA) qui représente un type de troubles neuro développementaux qui entravent la capacité à apprendre ou à utiliser les compétences académiques spécifiques (la lecture, l'écriture ou l'arithmétique). En plus, cette révision met l'accent sur la précision et la fluence en lecture, plutôt que sur la compréhension de lecture. Selon des statistiques internationales, ce trouble durable touche 5 à $8 \%$ des enfants scolarisés (Scerri \& SchulteKorne, 2010) et se manifeste lorsque l'enfant est confronté au langage écrit et ne parvient pas à identifier la plupart des mots de façon automatique. La dyslexie est indépendante de l'environnement social, culturel ou éducatif bien que ce dernier joue un rôle dans l'échec de l'apprentissage de la lecture. Outre les difficultés majeures en décodage, l'enfant dyslexique éprouve généralement une difficulté de prise de conscience de la structure sonore des mots, ou la conscience phonologique, qui est la capacité à distinguer et manipuler les sons dans les mots parlés de la langue, entraînant des difficultés au niveau de l'application des règles de conversion graphophonologique lors de la lecture (Bentin, 1992). 
Au-delà du rôle prépondérant reconnu de la conscience phonologique dans le développement de la lecture dans les différentes langues (Ziegler \& Goswami, 2005), il est également établi que la conscience phonologique peut être transférée d'une langue première à une autre et prédit ainsi la performance en lecture non seulement pour la langue maternelle, mais aussi pour la lecture en langue seconde (Al-Tamimi \& Rabab'ah, 2007 ; Durgunoğlu, 2002; Geva \& Wang, 2001; Saiegh-Haddad \& Geva, 2008). Le transfert phonologique peut être défini comme étant le processus par lequel les compétences de traitement phonologique, facilitent la lecture et / ou l'orthographe dans une autre langue. Le transfert des habiletés phonologiques d'une langue à une autre chez les enfants bilingues peut ainsi constituer un facteur prédictif de la lecture aussi bien en L1 qu'en L2 (Durgunoğlu, 2002; Saiegh-Haddad \& Geva, 2008 ; Koda, 2000).

Un vaste éventail de recherches ont en effet montré que la conscience phonologique est en étroite relation avec la conscience phonologique et avec la lecture de mots et de pseudo mots en L2 (Arab-Moghaddam \& Sénéchal, 2001). Ce constat indique que certaines compétences métalinguistiques développées en L1, peuvent être ajustées et appliquées lors de l'apprentissage de l'écrit en langue seconde, attestant du caractère transversal de ces compétences (Durgunoğlu, Nagy, \& Hancin-Bhatt, 1993). L'hypothèse du traitement central (Central Processing Hypothesis) reflète justement cette vision en postulant que l'acquisition de la lecture en L1 et en L2 dépend essentiellement des processus cognitifs linguistiques communs sous-jacents, y compris la conscience phonologique, et d'autres compétences cognitives, telles que la mémoire à court terme et la dénomination rapide (Durgunoğlu, Nagy, \& Hancin-Bhatt, 1993; Geva \& Siegel, 2000; Geva, Wade-Woolley, \& Shan, 1997). Cette hypothèse présume l'existence de niveaux similaires de conscience phonologique en L1 et L2, et, par conséquent, des niveaux comparables de précision de décodage des mots et des pseudo-mots dans les deux langues en question. Par conséquent, les difficultés observées au niveau d'une capacité cognitive-linguistique peuvent agir sur l'acquisition de la lecture dans les deux langues quelle que soit la complexité orthographique de la langue (Geva \& Siegel, 2000).

La question de savoir si les difficultés de lecture en L1 chez les enfants dyslexiques donnent toujours lieu à des problèmes similaires en L2, semble avoir suscité un grand intérêt dans la recherche sur la dyslexie et l'acquisition d'une langue seconde. Un grand nombre d'études ont montré que les sujets dyslexiques apprenant à lire une langue seconde, sont particulièrement faibles dans les compétences linguistiques phonologiques et syntaxiques à la fois dans L1 et L2, alors qu'ils ne présentent pas de difficultés majeures au niveau sémantique 
de la langue (Saiegh-Haddad, 2017). Ainsi, l'apprentissage de la lecture d'une langue seconde peut représenter un défi pour les enfants dyslexiques car les problèmes au niveau graphophonologique et de décodage peuvent entraver la possibilité de transfert de connaissances phonologiques de la langue maternelle à la langue seconde (Abu-Rabia \& Sammour, 2013; Høien \& Lundberg, 2000).

En outre, certaines recherches ont montré que le transfert phonologique est modulé par l'écart qui existe entre les deux structures linguistiques entre L1 et L2 (Pichette, Segalowitz, \& Connors, 2003). Ces différences structurelles entre L1 et L2, peuvent compliquer la lecture chez l'apprenant en difficulté de lecture (Besse, Demont, Gombert, 2007). Il est vrai que les recherches disponibles sur l'anglais L2, ont permis d'approfondir notre compréhension du transfert des connaissances phonologiques entre les systèmes orthographiques voisins (Comeau, Cormier, Grandmaison, \& Lacroix, 1999; Geva \& Siegel, 2000). Il n'en est toutefois pas ainsi pour les systèmes orthographiques structurellement très différents comme l'arabe et le français. Il convient donc d'élargir ce champ d'investigation à d'autres langues, en l'occurrence le français comme langue seconde chez les arabophones. Ainsi, la mise en relation d'une langue sémitique comme l'arabe (L1) et d'une langue indo-européenne comme le français (L2), permet de confronter deux systèmes d'écriture éloignés du point de vue de la consistance graphophonologique et la représentation phonologique.

Bien que les deux langues, l'arabe et le français, soient deux langues alphabétiques dans la mesure où elles sont basées sur la correspondance graphophonologique, ces deux orthographes utilisent des systèmes alphabétiques très différents auxquels s'ajoute une différence supplémentaire concernant la transcription du système vocalique. En outre, la langue arabe possède un système d'écriture spécifique et différent des langues indoeuropéennes, mais peu d'études sont réalisées sur les arabophones apprenant à lire le français langue seconde, bien que cette dernière occupe une place centrale dans la vie quotidienne dans certains pays comme l'Algérie, notamment dans le domaine éducatif où elle est considérée comme la première langue étrangère.

$\mathrm{Vu}$ le rôle primordial du facteur phonologique dans le développement de la compétence en lecture chez les enfants arabophones mis en évidence dans nombre d'études antérieures (Abu Rabia, 2001; Layes, Lalonde, Mecheri, \& Rebaï, 2015; Layes, Lalonde, \& Rebaï, 2015), et vu l'absence d'études examinant le rôle du facteur phonologique en Arabe L1 dans la performance de lecture en français L2 chez les enfants normo lecteurs en comparaison avec des sujets dyslexiques, la présente étude cherche à étudier le transfert des connaissances phonologiques, et son éventuel pouvoir prédictif dans l'apprentissage de la lecture. La 
question principale dans ce contexte est de savoir si les connaissances phonologiques acquises en arabe (L1) peuvent faciliter (ou non) l'apprentissage de la lecture en français (L2). Cette question peut être directement abordée en adoptant une approche comparative entre lecteurs normaux et dyslexiques du fait que le déficit phonologique représente le principal différenciateur entre ces deux groupes de lecteurs.

\section{Méthode}

\section{Participants}

Au total, 72 élèves (27 filles et 45 garçons) ont été sélectionnés des classes de $4^{\text {ème }}$ et $5^{\text {ème }}$ années primaires, répartis en deux groupes de lecteurs. Le groupe des lecteurs normaux est composé de 40 élèves, (24 garçons et 16 filles), d'âge moyen (130.44 mois ; écart type $=$ 7.03). Le groupe de dyslexiques $(n=32)$ comprend 13 filles et 19 garçons, la moyenne d'âge chronologique est de (129.60 mois; écart type $=8,73)$. L'aptitude mentale générale au test Raven a été prise en compte comme critère diagnostique d'inclusion pour le groupe dyslexique, montrant une performance adéquate par rapport aux sujets contrôles (Tableau 1).

Les sujets ayant une dyslexie qui ont participé à cette étude ont été préalablement présélectionnés par leurs enseignants, comme étant des élèves ayant des difficultés persistantes en lecture. Suite à la passation des tests de Raven et de lecture de mots et de pseudo mots, nous avons retenu 32 élèves parmi les 39 élèves initialement présélectionnés par les enseignants. Outre la passation des tests diagnostiques, nous nous sommes assuré que ces élèves sont indemnes de tous problèmes comportementaux, langagiers ou psychiques sur la base des livrets de santé scolaire.

Tous les enfants sont des locuteurs natifs de l'arabe dialectal, issus d'une classe socioéconomique moyenne. Tous les participants apprennent le français à l'école comme langue seconde. Conformément aux critères d'inclusion dans la dyslexie développementale, il était nécessaire de s'assurer que ces enfants sont exempts de tout déficit sensoriel, auditif ou visuel, et de tout autre problème susceptible d'avoir une influence sur leur compétence en lecture, et ce à travers l'examen des dossiers médico-scolaires et quelques entretiens avec le corps enseignant au sein des établissements scolaires où s'est déroulée la passation de nos tests.

Le consentement parental a été aussi obtenu au préalable avant la passation des tests par l'intermédiaire de l'administration de l'école ou par voie directe avec le chercheur. Ainsi, 
le formulaire de consentement destiné aux parents alphabètes décrit l'objectif de l'étude et les invitent à collaborer.

\section{Matériel et procédure}

\section{Test de raisonnement non verbal (Matrices Progressive de Raven)}

Les matrices progressives standard évaluent la capacité de raisonnement non verbal par analogie chez les enfants âgés de 7 à 11 ans et demi. Chaque item constitue un pattern avec une partie manquante. Six à huit images sont présentées aux deux côtés de l'item parmi lesquelles le sujet doit faire un choix pour compléter la partie manquante de manière adéquate. Chaque ensemble implique différents principes de transformation, et à l'intérieur de chaque ensemble, les items deviennent de plus en plus difficiles.

\section{Tests de lecture et de CP en Arabe (L1)}

Test de lecture des mots et des pseudo-mots $(\alpha=.89)$ : La lecture de mots et de pseudo-mots sont des aptitudes fondamentales liées au développement de la lecture dans les systèmes d'écriture alphabétiques. Ils sont des indices fiables pour les représentations orthographiques de mots chez l'enfant (Caravolas, 2018). Le test de lecture utilisé dans cette étude a été élaboré pour évaluer les compétences en lecture chez les enfants de 9-11 ans (Layes, Lalonde, Mecheri, \& Rebaï, 2015; Layes, Lalonde, \& Rebaï, 2015). Les participants ont pour tâche de lire à voix haute un ensemble de 80 stimuli présentés (40 mots fréquents et 40 mots non fréquents), sans aucune limite de temps. La catégorisation des mots selon le critère de fréquence est basée sur l'évaluation des enseignants du primaire en utilisant une échelle allant de 1 (mot près peu utilisé) à 4 (mot très fréquent). La tâche d'identification de pseudo-mots est constituée d'une liste de 20 items partiellement voyellisés et variés en longueur orthographique (nombre de syllabes).

Test de conscience phonologique $(\alpha=.70)$ : Ce test est composé de deux tâches : la suppression de syllabes (Layes, Lalonde, \& Rebaï, 2015) et la fusion de syllabes (Layes, Lalonde, Mecheri, \& Rebaï, 2015). La tâche de suppression de phonèmes est fréquemment utilisée pour l'évaluation de la conscience phonologique. Basé sur la présentation orale des mots (série de 20 mots), les participants ont pour tâche de supprimer (mentalement) soit la syllabe initiale, médiane ou finale du mot entendu, et de présenter ensuite oralement le segment restant de ce mot. La présence d'un déficit dans l'habileté de suppression de syllabes qui a été largement signalée dans la littérature, est considérée comme un facteur vigoureux de 
la dyslexie développementale. Dans ce test, deux essais sont administrés afin de s'assurer que les participants ont parfaitement compris la tâche à accomplir. Toutes ces tâches sont évaluées sur la base de l'exactitude des réponses.

Le test «fusion de phonèmes» est composé de dix paires de mots à présenter oralement à l'enfant qui doit isoler les syllabes initiales de chaque mot, puis prononcer la nouvelle combinaison obtenue de ces segments pris isolément. Les mots comprennent des groupes consonantiques bi-syllabiques.

\section{Tests de lecture et de CP en français (L2)}

Test de lecture de mots et de pseudo mots (coefficient test retest $=.64$ ). Choisis du manuel scolaire de français de $4^{\text {ème }}$ année de primaire, une liste de 40 mots est présentée aux participants pour la lecture à haute voix, dont 20 mots réguliers (exemples : soif, lune) et 20 autres mots irréguliers (exemples : sept, train). Les mots choisis sont appariés en termes de familiarité sur la base de l'avis des enseignants et en longueur (bi-syllabiques). La régularité orthographique fait référence dans ce contexte à la propriété qu'a le mot d'être prononcé comme il s'écrit (lettre par lettre), alors que les mots irréguliers ne requièrent pas la lecture lettre par lettre, pour des contraintes orthographiques spécifiques. Le choix de l'épreuve de lecture orale de mots réguliers et irréguliers (Laplante, 2003) répond au but d'évaluer la différence entre l'implication de la stratégie phonologique et la stratégie orthographique dans la reconnaissance des mots. Il est à noter que nombre de mots (réguliers et irréguliers) sont extraits de la liste de mots fréquents de l'Odédys (2002), et répondent au critère de familiarité mentionné plus haut, alors que les autres mots ne le font pas. En se basant sur le test de lecture de mots (réguliers et irréguliers) et de pseudo mots de l'Odédys (2002), nous avons changé quelques items sur la base de l'avis des enseignants questionnés sur la pertinence des items par rapport à leur familiarité à l'enfant.

La lecture de mots réguliers en français et la lecture de mots (fréquents et non fréquents) en arabe reposerait sur des processus quasiment similaires sur le plan phonologique, dans la mesure où il s'agit de la mise en œuvre d'une stratégie exclusivement phonologique. Cependant, la lecture de mots irréguliers en français nécessite, outre les habiletés de décodage, d'autres connaissances orthographiques spécifiques et particulièrement difficiles à exécuter chez les lecteurs en difficultés, puisque ces connaissances nécessitent une expérience suffisante avec la lecture. Ainsi, l'on s'attend à une corrélation significative entre, d'une part, la tâche phonologique et, d'autre part, la lecture de mots en arabe et la 
lecture de mots réguliers en français, et une association significative entre le décodage de pseudo mots en L1 et la lecture de mots irréguliers en L2.

Extraits du test Odédys (2002), vingt pseudo mots sont appariés selon à la longueur et à la structure phonémique avec les mots réguliers utilisés dans ce test. Les sujets sont d'abord avertis de la nature des items avant la lecture de la liste, et sont ensuite invités à lire à haute voix les items successivement.

\section{Test d'évaluation de la conscience phonologique}

L'objectif est de vérifier si le déficit de la conscience phonologique en L1, caractérisant l'enfant dyslexique, se retrouve en L2 chez les mêmes participants. Le test (Odédys, 2002) consiste à supprimer des phonèmes après présentation orale de mots à chaque participant, qui doit supprimer le phonème initial du mot qu'il entend, et énoncer ensuite la syllabe restante (exemple: « flou» devient «lou»). Ce subtest est choisi pour la facilité de cette épreuve qui demande de retenir temporairement un seul mot au lieu de deux mots comme dans le subtest de fusion de phonèmes.

\section{Résultats}

\section{Comparaisons des performances entre lecteurs normaux et dyslexiques}

Les données statistiques descriptives sont présentées dans le tableau (1). Comme attendu, les enfants ayant une dyslexie ont montré une insuffisance dans les mesures de lecture de mots et de pseudo mots et de conscience phonologique en L1 comme en L2.

Tableau 1. Performances des lecteurs normaux (LN) et des dyslexiques (Dys) dans tous les tests de lecture et de conscience phonologique (L1 et L2)

\begin{tabular}{llll}
\hline \multirow{2}{*}{ Tests } & LN $(\mathrm{n}=40)$ & Dys $(\mathrm{n}=32)$ & \\
\cline { 2 - 4 } & $\mathrm{M}(\mathrm{ET})$ & $\mathrm{M}(\mathrm{ET})$ & $\mathrm{T}(2.00)$ \\
\hline Age (en mois) & $130.38(7.25)$ & $129.63(8,60)$ & $.40 \mathrm{~ns}$ \\
Raven & $17.65(3.97)$ & $16.72(1.90)$ & $1.21 \mathrm{~ns}$ \\
Pseudo mots L1 & $16.83(6.29)$ & $6.03(3.64)$ & $9.10^{* * *}$ \\
Lecture de mots L1 & $57.03(7.15)$ & $36.97(5.64)$ & $12.94 * * *$ \\
Total lecture L1 & $73.85(12.35)$ & $43.00(6.65)$ & $13.52 * * *$ \\
CP suppression L1 & $10.85(3.35)$ & $8.63(3.49)$ & $2.74 * *$ \\
CP fusion L1 & $10.35(4.19)$ & $6.19(3.64)$ & $4.43 * * *$
\end{tabular}




\begin{tabular}{llll} 
Total CP L1 & $21.20(5.83)$ & $14.81(6.60)$ & $4.35^{* * *}$ \\
Mots réguliers L2 & $1.33(1.26)$ & $0.69(1,09)$ & $2.29^{*}$ \\
Mots irréguliers L2 & $0.68(1.11)$ & $0.13(0.33)$ & $2.94^{* *}$ \\
Total mots L2 & $22.00(1,96)$ & $0.69(1.09)$ & $3.59^{* * *}$ \\
Pseudo mots L2 & $7.75(3.77)$ & $2.06(1.75)$ & $8.45^{* * *}$ \\
CP L2 & $3.18(2.09)$ & $1.38(1.15)$ & $4.21^{* * *}$ \\
\hline
\end{tabular}

Note $: \mathrm{CP}=$ conscience phonologique; $* \mathrm{P}<.05 ; * * \mathrm{P}<.01 ; * * * \mathrm{P}<.001 ;$ n.s. $=$ non significatif.

Une analyse multiple de covariance (MANCOVA) a été effectuée avec toutes les mesures de lecture et de CP en L1 (arabe) et en L2 (français) comme variables dépendantes, avec le "groupe" de lecteurs comme facteur fixe et l'âge des participants comme covariant. On constate un effet principal du facteur "groupe" sur ces variables dépendantes (la trace de Pillai $\left.=.71, \mathrm{~F}_{(8.62)}=19.41, \mathrm{p}<.000, \eta 2=.71\right)$.

Étant donné le niveau de signification de l'indice de Pillai pour l'effet de groupe, nous avons procédé à l'examen des effets univariés potentiels. Des valeurs significatives d'ANOVA ont été obtenues indiquant des effets principaux pour les variables indépendantes suivantes: la lecture de mots $\left(\mathrm{F}_{(1.69)}=152.83, \mathrm{p}<.000, \eta 2=.63\right.$; la taille d'effet $\left.=1\right)$; la lecture de pseudo mots $\left(\mathrm{F}_{(1.69)}=67.83, \mathrm{p}<.000, \eta 2=.49\right.$; la taille d'effet $\left.=1.00\right)$; la CP $(\mathrm{F}$ ${ }_{(1.69)}=13.00, \mathrm{p}<.000, \eta 2=.19$; la taille d'effet $\left.=.97\right)$; la lecture de mots en L2 (F (1.69) $=$ 7.34, $\mathrm{p}<.008, \eta 2=.09$; la taille d'effet $=.76)$; la lecture de pseudo mots en L2 (F (1.69) $=$ 55.01, $\mathrm{p}<.000, \eta 2=.49$; la taille d'effet $=1.00$ ), et finalement la conscience phonologique en L2 $\left(\mathrm{F}_{(1.69)}=14.64, \mathrm{p}<.000, \eta 2=.17\right.$; la taille d'effet $\left.=.96\right)$. 


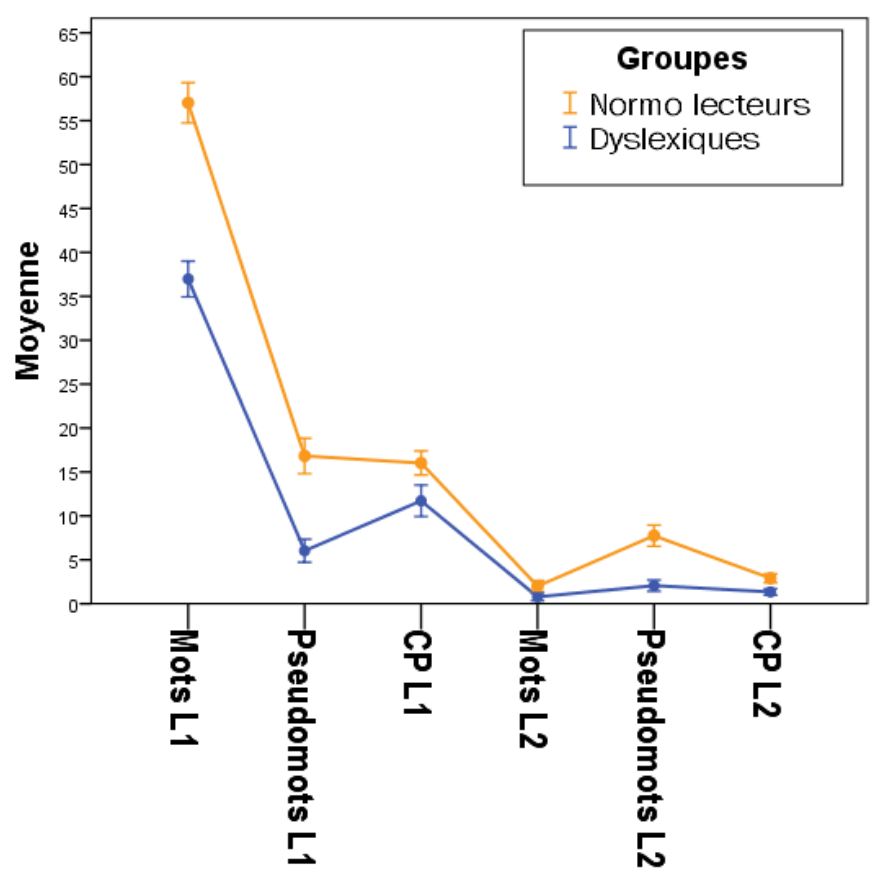

Figure 1. Comparaisons entre normo lecteurs et dyslexiques dans les tâches de lecture et de CP en L1 et L2

\section{Corrélations entre la lecture et la conscience phonologique en L1 et L2}

En vue d'examiner les relations entre la lecture (mots et pseudo-mots) et la conscience phonologique en L1et en L2, une analyse de corrélation a été effectuée pour l'ensemble des participants (Tableau 2). Les résultats montrent une corrélation positive très significative entre les différentes mesures, sauf pour la CP L1et la lecture de mots en L1 qui sont modérément corrélées.

Il est à noter que le Tableau 2 montre qu'aucune valeur de corrélation n'atteint la limite de 0.8 , à l'exception des pseudo-mots en L1 et L2, indiquant que qu'aucun problème de colinéarité se pose.

Tableau 2. Corrélations entre toutes les mesures pour l'ensemble des participants $(n=72)$

\begin{tabular}{|c|c|c|c|c|c|c|}
\hline Mesures & 1 & 2 & 3 & 4 & 5 & 6 \\
\hline 1. Total mots L1 & - & $.79 * * *$ & $.52 * * *$ & $.41^{* * *}$ & $.76^{* * *}$ & $.59^{* * *}$ \\
\hline 2. Pseudo mots L1 & & - & $.36^{* * *}$ & $.54 * * *$ & $.95^{* * *}$ & $.55^{* * *}$ \\
\hline 3. CP L1 & & & - & $.28^{* *}$ & $.44 * * *$ & $.64 * * *$ \\
\hline 4. Total mots L2 & & & & & $.57 * * *$ & $.46^{* * *}$ \\
\hline 5. Pseudo mots L2 L2 & & & & & - & $.58^{* * *}$ \\
\hline 6. CP L2 & & & & & & - \\
\hline
\end{tabular}

Note. $* * p<.01 ; * * * P<.001$ 


\section{La prédiction de la performance en lecture en $L 1$ et en $L 2$}

En vue de déterminer les variables les plus prédictives des performances de lecture en L2, en accordant aux scores des élèves à l'épreuve de lecture de mots écrits en L2 le statut de variable dépendante et les mesures de décodage de pseudo mots et la CP en L1 le statut de variables explicatives. Ces facteurs ont été introduits respectivement dans le modèle de régression pas à pas pour chaque groupe de lecteurs séparément. Comme le montre le tableau (3) les résultats indiquent que les variables contribuant le plus à expliquer la variabilité des scores de lecture de mots en L2 chez les lecteurs normaux, sont celles mesurées par le décodage de pseudo mots (L1) qui apporte la contribution la plus importante (41\%) suivie par la CP en L1 avec (22\%). Chez le groupe de dyslexiques, la CP explique (32\%) de la variance de lecture de mots L2 alors que le décodage de pseudo mots en contribue seulement à (9\%).

Tableau 3. Analyse de régression pas à pas pour la performance de lecture de mots en L2 pour chaque groupe de lecteur séparément

\begin{tabular}{llllll}
\hline Groupe & Variables & & & \\
& indépendantes & $\beta$ & $\mathrm{R}^{2}$ & $\mathrm{R}^{2}$ ajusté & $\mathrm{F}$ \\
\hline \multirow{2}{*}{ Lecteurs Normaux } & Pseudo mots L1 & .56 & .42 & .41 & $28.42 * * *$ \\
& CP L1 & .48 & .65 & .63 & $24.50^{* * *}$ \\
\hline \multirow{2}{*}{ Dyslexiques } & CP L1 & -.61 & .34 & .32 & $15.62 * * *$ \\
& Pseudo mots L1 & -.32 & .44 & .41 & $5.47 *$ \\
\hline
\end{tabular}

Note: $* \mathrm{P}<.05 ; * * * \mathrm{P}<.001$

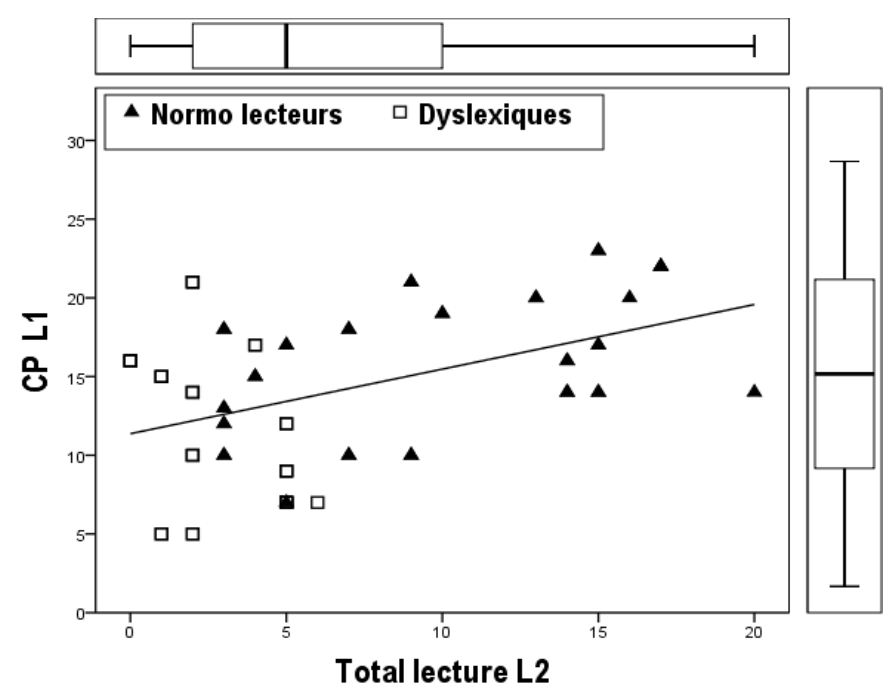

Figure 2. Régression des scores de CP L1 et la lecture de mots et de pseudo mots en L2 


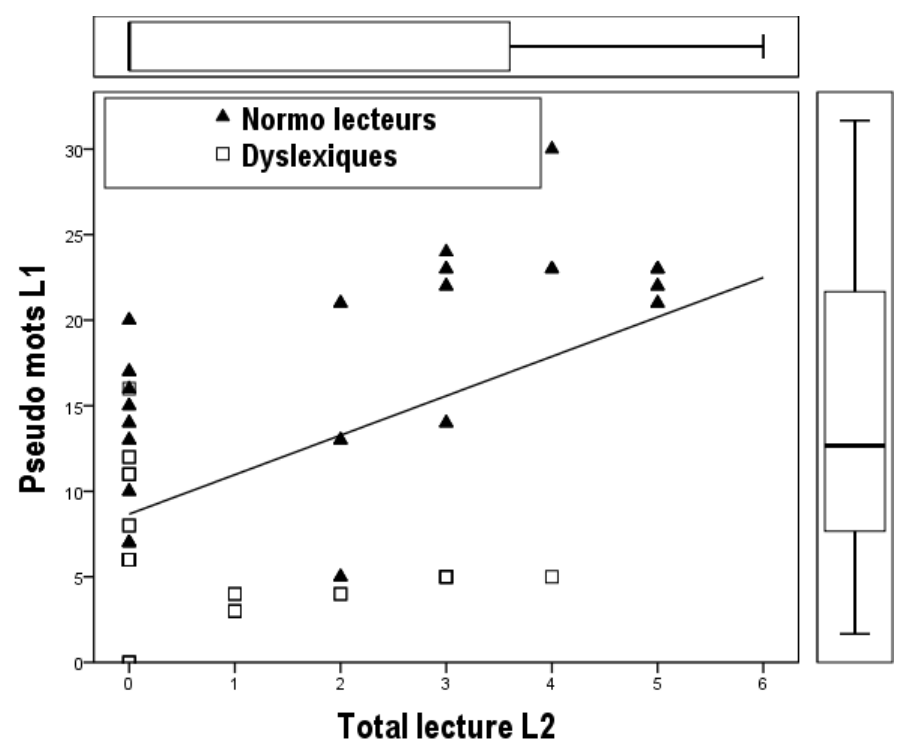

Figure 3. Régression des scores de pseudo mots L1 et de la lecture de mots et pseudo mots en L2

\section{Discussion}

La principale question qui a guidé cette étude était de savoir si la conscience phonologique en arabe (L1) influe la performance de lecture en français (L2) chez des enfants arabophones. Ce pattern de prédiction pourrait être expliqué en termes de transfert de connaissances phonologiques entre L1 et L2. D'abord, en comparant les scores des deux groupes de lecteurs normaux et dyslexiques dans les différentes tâches de lecture et phonologiques, nos résultats montrent des différences significatives entre les deux groupes de lecteurs dans toutes les mesures de lecture et de CP en L1 et en L2. Ces différences indiquent, outre la présence de déficit phonologique et de décodage en L1, une faiblesse similaire marquante dans le décodage et de la CP en L2 chez les sujets souffrant d'une dyslexie développementale (Oren \& Breznitz, 2005).

Cependant, les scores élevés obtenus en lecture en arabe (L1) par rapport à la lecture en français (L2) chez le groupe de normo lecteurs, sont vraisemblablement liés au niveau de compétence linguistique orale en L1, impliquant essentiellement les connaissances phonologiques (Geva et al., 1997). Ainsi une faible conscience phonologique en L2 qu'en L1 peut être attribuée à une faiblesse comparable en langue orale L2 (Saiegh-Haddad, 2017). Les représentations phonologiques des mots en L2 pourraient être moins matures que les représentations phonologiques des mots en L1, car ces derniers ont été acquis à un âge plus précoce et sont ainsi plus familiers à l'enfant. Néanmoins, l'ampleur du lexique en L1 peut aussi avoir un impact sur la compétence phonologique en L1 à travers l'augmentation des 
compétences linguistiques générales telles que la capacité à traiter sélectivement les caractéristiques phonologiques des mots.

Les résultats de cette étude sont concluants vu l'association significative révélée à travers l'analyse de corrélation entre la CP et le décodage de mots et de pseudo mots en L1 d'une part, et la lecture de mots (réguliers et irréguliers) et de pseudo mots et la CP en L2, indiquant que la CP est un facteur cognitif plutôt qu'un facteur spécifique au langage (WadeWoolley \& Geva, 2000). Ces résultats sont en accord avec ceux de Saiegh-Haddad et Geva (2008), qui ont exploré la relation entre la conscience phonologique et morphologique chez des sujets bilingues anglais-arabe, en essayant de comprendre si ces compétences métalinguistiques sont liées à la précision de lecture des mots et de pseudo mots. Les résultats ont montré que les deux langues sont significativement et positivement corrélées. Ils ont également constaté que la CP est constamment corrélée avec la lecture des mots et des pseudo-mots quelle que soit la langue, tandis que la conscience morphologique ne l'est pas.

L'analyse de régression a été menée pour déterminer si la compétence phonologique reliée à la lecture peut être transférée de la L1 à la L2 dans l'apprentissage de la lecture (Chung \& Ho, 2010). Les résultats d'analyse de régression pas à pas montrent que la $\mathrm{CP}$ et la lecture de pseudo mots en L2 prédisent la lecture de mots en L1 pour les lecteurs normaux et les dyslexiques, ce qui indique que les connaissances phonologiques acquises, quel que soit le niveau atteint de performance de lecture, pourraient faire l'objet d'un transfert de L1 en L2, permettant ainsi aux lecteurs d'acquérir un niveau correspondant de connaissances de lecture en L2.

Ces données appuient l'idée que les processus phonologiques fondamentaux sousjacents à la lecture en L1 le sont vraisemblablement pour la lecture en L2. Ces processus fondamentaux en L1 sont susceptibles d'être de prédicteurs robustes de la lecture de mots en L2. Pratiquement, de tels résultats suggèrent qu'une détection précoce des compétences reliées à la lecture de L1 pourrait également aider à identifier les lecteurs à risque qui pourraient avoir des difficultés dans les compétences en L2.

Des résultats similaires ont été rapportés par Alshaboul et al. (2014) montrant que la conscience phonologique en arabe (L1) apporte une contribution de 50\% à la prédiction de la lecture mots en anglais (L2) et $19 \%$ pour les pseudo-mots. Ces résultats indiquent la transférabilité de la conscience phonologique de (L1) à (L2) suggérant que les enfants ayant une bonne connaissance phonologique en arabe sont plus susceptibles de devenir de meilleurs lecteurs en anglais. Ceci est cohérent avec d'autres études montrant la présence de transfert inter-langues de la compétence phonologique qui prédit le développement de la lecture dans la 
langue seconde même si les deux langues sont différentes en termes de système orthographique (Gottardo, Stanovich, \& Siegel, 1996) suggérant la présence d'un mécanisme de traitement de la langue universelle (Cummins, 1989). La capacité d'appliquer des connaissances stratégiques du traitement phonologique de L1 à L2 semble donc se produire indépendamment de la différence des deux systèmes d'écriture comme le français et l'arabe (Wagner, Spratt, \& Ezzaki, 1989).

Plusieurs travaux sur le transfert de compétences linguistiques ont montré que les habiletés phonologiques acquises en langue maternelle sont des prédicteurs tangibles de la capacité de lecture dans une autre langue seconde (Wade-Woolley \& Geva, 2000). Durgunoglu et ses collaborateurs (Durgunoğlu et al., 1993), ont étudié un groupe d'hispanophones apprenant à lire en anglais (L2), et ont montré que les connaissances et les habiletés acquises en L1 peuvent être transférées lors de l'apprentissage d'une langue seconde (L2). En revanche, puisque la conscience phonologique est hautement impliquée dans l'acquisition de la lecture, les apprentis lecteurs d'une langue seconde, qui doivent restructurer leurs connaissances phonologiques, sont plus susceptibles d'éprouver des difficultés lors de ce processus d'acquisition.

Nos résultats sont dans la lignée de nombreuses études ayant examiné le transfert de compétences phonologiques entre l'arabe (dans sa forme voyellisée) et l'anglais (Abu-Rabia \& Siegel, 2002 ; Abu-Rabia \& Siegel, 2003), qui ont souligné la présence d'un transfert de compétences phonologiques entre l'arabe et l'anglais, et ont également trouvé une corrélation significative entre la conscience phonologique en arabe, et l'acquisition de la lecture en anglais. Ces résultats sont en accord avec Alshaboul et ses collaborateurs (Alshaboul, Asassfeh, Alshboul, \& Alodwan, 2014) qui ont montré l'existence de transfert phonologique de l'arabe (L1) vers l'anglais (L2) chez des sujets normo lecteurs bilingues âgés de 6 à 10 ans. D'autres études ont également montré que les compétences phonologiques peuvent être transférées à travers les langues, et peuvent prédire par conséquent les difficultés de lecture dans une autre langue en tenant compte des différences orthographiques (Abu-Rabia \& Siegel, 2003).

Des résultats similaires ont été rapportés par Wade-Woolley et Geva (2000) qui ont examiné le transfert linguistique des connaissances phonologiques entre l'anglais et l'hébreu (orthographe transparente), chez les enfants bilingues apprenant à lire dans les deux langues. Ce constat indique l'importance des relations entre les systèmes phonologiques en termes de principes phonologiques. 
Nos résultats soutiennent le point de vue de Wagner, Spratt et Ezzaki (1989), selon lequel la conscience phonologique peut être transférée entre la L1 et la L2 même si les deux systèmes orthographiques ne sont pas structurellement comparables, comme dans le cas de l'arabe et du français. Ce constat corrobore «l'hypothèse de l'interdépendance linguistique » de Cummins (1989) suggérant que la maîtrise d'une langue première peut faciliter l'apprentissage de nouvelles langues, et que la présence d'un déficit en L1 peut causer un déficit correspondant en L2.

Le transfert inter-langues des compétences phonologiques a été attribué à des éléments linguistiques généraux impliqués dans les tâches de conscience phonologique et qui relèvent de plusieurs domaines, comprenant les exigences auditives, perceptuelles, cognitives, attentionnelles et métalinguistiques. Le transfert peut également être attribué à des éléments spécifiques à la langue, en fonction du degré de similarité des langues dans les domaines phonologique et orthographique (Wade-Woolley \& Geva, 2000).

D'après Saiegh-Haddad (2017), la conscience phonologique en L2 n'est pas une fonction purement métalinguistique se rapportant à une compétence cognitive générale. Elle est plutôt fortement influencée par les représentations phonologiques dans la mémoire à long terme qui sont liées au langage oral, et par la distance phonologique entre L1 et L2. Ces données soulignent le rôle principal des facteurs linguistiques dans la conscience phonologique conçue, comme étant une construction bidimensionnelle basée sur des représentations phonologiques installées dans la mémoire à long terme.

Concernant le caractère universel du facteur phonologique à travers les différentes langues, considéré comme un traitement central dans le développement de la lecture, une présomption assez répandue tend à considérer que les mêmes habiletés cognitives et linguistiques sous-jacentes à l'apprentissage de la lecture de la langue première (L1) sont indispensables à l'apprentissage de la lecture dans une langue seconde (L2). Ce qui signifie que cette capacité phonologique centrale (la conscience phonologique), développée avant et au cours du développement de la L1, influe sur le développement des compétences en lecture dans un environnement linguistique bilingue.

Le résultat principal de cette étude évoque la possibilité de transférabilité des connaiisances phonologiques en arabe (L1) à la lecture de mots en français (L2), indiquant la présence d'un transfert positif entre ces deux langues. Cela pourrait être expliqué par le fait que la conscience phonologique est significativement corrélée avec les performances de lecture chez les participants. Ceci suggère que le pouvoir qu'à la conscience phonologique pour prédire la performance en lecture de mots. 


\section{Conclusions}

La présente étude portait sur la question de la place centrale du transfert des connaissances phonologiques entre lecture en arabe (L1) et en français (L2). Les résultats de cette étude offrent un argument assez clair en faveur de la présence de transfert positif de connaissances phonologiques entre une langue maternelle transparente (l'arabe voyellisé) et une langue seconde (le français), suggérant qu'un enseignement bilingue pourrait être envisageable très précocement chez l'enfant arabophone.

De tels résultats devraient sensibiliser la communauté éducative sur la question de l'apprentissage des langues secondes par les élèves ayant des difficultés d'apprentissage de la lecture. Elle encourage la mise en place de programmes éducatifs visant à les impliquer dans l'apprentissage explicite de la conscience phonologique en L1. L'impact positif attendu de tels programmes d'entrainement phonologique sur l'apprentissage de la lecture en L2, peut, à son tour, se répercuter positivement sur le développement de la lecture en L1 sur la base du « transfert rétroactif ». Selon l'hypothèse de transfert cognitif rétroactif (Cognitive Retroactive Transfer, CRT), le transfert de connaissances métalinguistiques et phonologiques en particulier, s'effectuerait réversiblement, c'est à dire de la L2 à la L1.

Cependant, le rôle des expériences préalables de l'écrit sur le développement des connaissances et l'apprentissage de la lecture en français L2 paraît important comme l'ont souligné Besse et al. (2007), même si l'on reconnait le rôle d'autres facteurs linguistiques comme la familiarité avec la langue seconde. Il est donc nécessaire d'approfondir les résultats en essayant de mieux tenir compte les conditions d'apprentissage de la lecture en français L2. Des recherches ultérieures visant à approfondir ces résultats pourraient s'intéresser davantage à l'étude des différences entre l'arabe et le français dans la mise en œuvre des connaissances métalinguistiques plus générales, en particulier morphologiques, avec ou sans interaction avec le facteur phonologique.

En somme, les enfants qui ont des scores elevés dans la conscience phonologique en arabe ont plus de chances de réussir l'apprentissage de la lecteur en francais. Ce constat peut impacter considérablement le processus d'apprentissage de la lecture en langue seconde dans le contexte linguistique arabophone. Ainsi, les instructeurs peuvent utiliser les connaissances phonologiques en L1 (l'arabe) pour appuyer l'apprentissage de lecture en L2. Ceci est valable pour les lecteurs normaux et pour les enfants ayant une difficultés en lecture voire dyslexiques. 


\section{Références}

Abu-Rabia, S., \& Sammour, R. (2013). Spelling errors' analysis of regular and dyslexic bilingual Arabic-English students. Journal of Modern Linguistics, 3, 58-68. DOI: https://doi.org/10.4236/ojml.2013.31007

Abu-Rabia, S., \& Siegel, L.S. (2002). Reading, syntactic, orthographic, and working memory skills of bilingual Arab Canadian children. Journal of Psycholinguist Research, 31, 661-678. DOI: https://doi.org/10.1007/bf01026951

Abu-Rabia, S., \& Siegel, L.S. (2003). Reading and writing skills in three orthographies. Reading and Writing, 16, 611- 634.

Abu-Rabia, S. (2001). The role of vowels in reading Semitic scripts: Data from Arabic and Hebrew. Reading and Writing, 14, 39-59.

Alshaboul, Y., Asassfeh, S., Alshboul, S., \& Alodwan, T. (2014). The contribution of L1 phonemic awareness into L2 reading: The case of Arab EFL readers. International Education Studies, 7, (3), 99-111. DOI: https://doi.org/10.5539/ies.v7n3p99

Al-Tamimi, Y., \& Rabab'ah, G. (2007). The relationship between phonological awareness and word reading. Poznan Studies in Contemporary Linguistics, 43, 5-21. DOI: https://doi.org/10.2478/v10010-007-0011-6

American Psychiatric Association (2000). Diagnostic and Statistical Manual of Mental Disorders, 4th ed. Washington D.C.: American Psychiatric Association.

American Psychiatric Association (2013). Diagnostic and statistical manual of mental disorders, 5th ed. Arlington: American Psychiatric Association.

Arab-Moghaddam, M., \& Sénéchal, M. (2001). Orthographic and phonological processing skills in reading and spelling in Persian/English bilinguals. International Journal of Behavioural Development, 25, 140-147. DOI: https://doi.org/10.1080/01650250042000320

Besse, A.S., Demont, E., \& Gombert, J.E. (2007). Traitements phonologiques et morphologiques dans l'apprentissage de la lecture en français langue seconde par des arabophones et des lusophones. Psychologie Française, 52 (1), 89-105.

Boudelaa, S., \& Marslen-Wilson, W.D. (2010). ARALEX: A lexical database for Modern Standard Arabic. Behaviour Research Methods, 42, 481-487. DOI: https://doi.org/10.3758/brm.42.2.481 
Caravolas, M. (2018). Growth of word and pseudoword reading efficiency in alphabetic orthographies: Impact of consistency. Journal of Learning Disabilities, 51 (5), $422-$ 433. DOI: https://doi.org/10.1177/0022219417718197

Chung, K-K-H., \& Ho, C-S-H. (2010). Second language learning difficulties in Chinese children with dyslexia: what are the reading-related cognitive skills that contribute to English and Chinese word reading? Journal Learning Disabilities, 43 (3), 195-211. DOI: https://doi.org/10.1177/0022219409345018

Comeau, L., Cormier, P., Grandmaison, A.R., \& Lacroix, D. (1999). A longitudinal study of phonological processing skills in children learning to read in a second language. Journal Educational Psychology, 91 (1), 29-43. DOI: https://doi.org/10.1037//0022$\underline{0663.91 .1 .29}$

Cummins, J. (1989). A theoretical framework for bilingual special education. Exceptional Child, 56, 111-119. DOI: https://doi.org/10.1177/001440298905600203

Durgunoğlu, A.Y., Nagy, W.E., \& Hancin-Bhatt, B.J. (1993). Cross-language transfer of phonological awareness. Journal Educational Psychology, 85, 453-465.

Durgunoğlu, A.Y. (2002). Cross-linguistic transfer in literacy development and implications for language learners. Annals of Dyslexia, 52, 189-204. DOI: https://doi.org/10.1007/s11881-002-0012-y

Geva, E., \& Siegel, S. (2000). Orthographic and cognitive factors in the concurrent development of basic reading skills in two languages. Reading and Writing, 21, 1-30.

Geva, E., Wade-Woolley, L., \& Shany, M. (1997). Development of reading efficiency in first and second language. Scientific Studies on Reading, 1 (2), 119-144. DOI: https://doi.org/10.1207/s1532799xssr0102_2

Geva, E., \& Wang, M. (2001). The development of basic reading skills in children: A crosslanguage perspective. Annual Review Applied Linguistics, 21, 182-204. DOI: https://doi.org/10.1017/s0267190501000113

Gottardo, A., Stanovich, K.E., \& Siegel, L.S. (1996). The relationships between phonological sensitivity, syntactic processing, and verbal working memory in the reading performance of third-grade children. Journal of Experimental Child Psychology, 63, 3, 563-582. DOI: https://doi.org/10.1006/jecp.1996.0062

Høien, T., \& Lundberg, I. (2000). Dyslexia: From theory to intervention. Dordrecht: Kluwer Academic Publishers.

Koda, K. (2000). Cross-linguistic variations in L2 morphological awareness. Applied Psycholinguistics, 21 (3), 297-320. DOI: https://doi.org/10.1017/s0142716400003015 
Laboratoire Cogni-Sciences IUFM de Grenoble (2002). Outil de dépistage des dyslexies (Odédys). Grenoble: Laboratoire Cogni-Sciences IUFM de Grenoble.

Laplante, L. (2002). Épreuve de lecture orale de mots réguliers et irréguliers, non-publiée. Dans M. Cloutier et A. Leclerc (eds.), Apprentissage de la lecture chez des arabophones et des francophones identifiés à risque du premier cycle du primaire. Aux grands mots les grands travaux. Récupéré de http://www.er.uqam.ca/nobel/scilang/cesla04/pdf/lecture.pdf

Layes, S., Lalonde, R., Mecheri, S., \& Rebaï, M. (2015). Phonological and cognitive reading related skills as predictors of word reading and reading comprehension among Arabic dyslexic children. Psychology, 6, 20-38. DOI: https://doi.org/10.4236/psych.2015.61003

Layes, S., Lalonde, R., \& Rebaï, M. (2015). Reading speed and phonological awareness deficits among Arabic-speaking children with dyslexia. Dyslexia, 21 (1), 80-95. DOI: https://doi.org/10.1002/dys.1491

Oren, R., \& Breznitz, Z. (2005). Reading processes in L1 and L2 among dyslexic as compared to regular bilingual readers: Behavioural and electrophysiological evidence. Journal of Neurolinguistics, 18, 127-151. DOI: https://doi.org/10.1016/j.jneuroling.2004.11.003

Pichette, F., Segalowitz, N., \& Connors, K. (2003). Impact of maintaining L1 reading skills on L2 reading skill development in adults: Evidence from speakers of Serbo-Croatian learning French. Modern Language Journal, 87 (3) 391-403. DOI. https://doi.org/10.1111/1540-4781.00197

Saiegh-Haddad, E., \& Geva, E. (2008). Morphological awareness, phonological awareness, and reading in English-Arabic bilingual children. Reading and Writing, 21, 481-504. DOI: https://doi.org/10.1007/s11145-007-9074-X

Saiegh-Haddad, E. (2017). What is phonological awareness in L2? Journal of Neurolinguistics. DOI: https://doi.org/10.1016/j.jneuroling.2017.11.001

Scerri, T.S., \& Schulte-Korne, G. (2010). Genetics of developmental dyslexia. European Child and Adolescence Psychiatry, 19, 179-197.

Wade-Woolley, L., \& Geva. E. (2000). Processing novel phonemic contrasts in the acquisition of L2 word reading. Scientific Studies of Reading, 4, 295-311. DOI: https://doi.org/10.1207/s1532799xssr0404_3 
Wagner, D.A., Spratt, J.E., \& Ezzaki, A. (1989). Does learning to read in a second language always put the child at a disadvantage? Some counterevidence from Morocco. Applied Psycholinguistics, 10, 31-48. DOI: https://doi.org/10.1017/s0142716400008407

Ziegler, J.C., \& Goswami, U. (2005). Reading acquisition, developmental dyslexia, and skilled reading across languages: a psycholinguistic grain size theory. Psychological Bulletin, 131 (1), 3-29. DOI: https://doi.org/10.1037/0033-2909.131.1.3

\section{Informations sur les auteurs}

Smail Layes, détenteur d'un doctorat en orthophonie, exerce la fonction de professeur au département de psychologie à l'université d'El-Oued. Il est aussi titulaire d'un doctorat en psychologie de l'université de Rouen. Ses travaux de recherche porte essentiellement sur la dyslexie, la dysgraphie et la dyscalculie chez l'enfant.

E-mail: smail.layes@gmail.com

Kamel Layes, détenteur de diplome d'étude spécialisée en soins psychaitriques, est professionnel de la santé mentale à l'hopital d'El-Oued depuis plus de 20 ans. Il est également détenteur de licence et de master en "difficultés d'apprentissage" de l'université d'El-Oued. Actuellement il est doctorant en "éducation spécialisée" à l'université d'Alger 2.

E-mail: kamellayes65@gmail.com

Khenfour Hichem, est directeur du "Centre des Enfants Assistés" à El-Oued. Il est également détenteur de licence et de master en "difficultés d'apprentissage" de l'université d'El-Oued, et il est actuellement doctorant en “difficultés d'apprentissage" à l'université d'El-Oued.

E-mail: hichemm70@gmail.com

Pour citer cet article:

Layes, S., Layes, K., \& Khenfour, H. (2018). Le rôle de la conscience phonologique dans l'apprentissage de la lecture en français langue seconde par les enfants arabophones : comparaison entre lecteurs normaux et dyslexiques. Bellaterra Journal of Teaching \& Learning Language \& Literature, 11(4), 21-40. DOI: https://doi.org/10.5565/rev/jt13.775 